Technical Report No. 2/04, June 2004

REGIME-SWITCHING PERIODIC MODELS FOR CLAIM COUNTS

Yi Lu and José Garrido 


\title{
Regime-Switching Periodic Models for Claim Counts
}

\author{
Yi Lu* ${ }^{1 *}$ and José Garrido ${ }^{2}$ \\ Department of Mathematics and Statistics \\ Concordia University, Canada
}

\begin{abstract}
We study a Cox risk model that accounts for both, seasonal variations and random fluctuations in the claims intensity. This occurs with natural phenomena that evolve in a seasonal environment and affect insurance claims, such as hurricanes.

More precisely, we define an intensity process, governed by a periodic function with a random peak level. The periodic intensity function follows a deterministic pattern in each short-term period, and is illustrated by a beta-type function. A two-state Markov chain defines the level process, explaining the random effect due to "high" or "low risk" years. This yields a regime-switching process, alternating between the two resulting intensities.

The properties of the corresponding claim counting process are discussed in detail. By properly defining the Lundberg coefficient, Lundberg-type bounds for finite time ruin probabilities are derived.
\end{abstract}

Keywords: Cox model; Non-homogeneous Poisson process; Regime-switching process; Periodicity; Lundberg-type bound; Ruin probability; Risk theory

${ }^{*}$ This research was funded by a ${ }^{1}$ Society of Actuaries PhD Grant, a Post-Graduate Scholarship and ${ }^{2}$ operating grant OGP0036860 of the Natural Sciences and Engineering Council of Canada (NSERC). 


\section{Introduction}

Consider the risk process

$$
U(t)=u+c t-\sum_{j=1}^{N(t)} X_{j}, \quad t \geq 0,
$$

where $u$ is the initial value, $c$ is the (constant) premium rate, $\{N(t)\}_{t \geq 0}$ is a point process which models the number of claims arriving within the time interval $[0, t)$ and $X_{j}$ is the $j$-th claim size. When $\{N(t)\}_{t \geq 0}$ is a Poisson process with (constant) intensity $\lambda$ and the claim size sequence $\left\{X_{j}\right\}_{j \geq 1}$ are i.i.d. and independent of $N$, then (1) is known as the classical (homogeneous Poisson) risk model, which has been investigated extensively in the actuarial literature.

The classical risk model is not realistic in some practical situations. Two main modifications are made here. First, a non-homogeneous Poisson (NHP) process is used to model "size fluctuations" in the claim intensity of a risk subject to seasonality. Then, a Cox process, also called doubly stochastic Poisson process and a natural extension of the NHP process, is used to characterize the underlying "risk fluctuations" in the claims intensity [see Grandell (1991)].

The risk theory literature gives only a few results when the claim counting process is a NHP process. Dassios and Embrechts (1989) defines a risk model with periodic claim intensity and consider the corresponding ruin problems using a martingale approach. Similar models are also considered by Asmussen and Rolski (1994) and by Rolski et al. (1999). Two-sided bounds and asymptotic formulae for ruin probabilities are derived by using an average arrival rate risk model. Berg and Haberman (1994) uses a non-homogeneous Markov birth process, of which the NHP process is a special case, to predict trends in life insurance claim occurrences. Dimitrov et al. (2000) exploits some properties in a NHP risk model with a (single) periodic claim intensity. Morales (2004) chooses a Gaussian shape for the periodic claim intensity function. By contrast, Garrido and Lu (2002) considers a more general double periodic intensity rate. Possible forms of intensity functions, like the double-beta and the sine-beta, are proposed.

An early reference to Cox risk models is Ammeter (1948). In his model, the intensity $\lambda_{k}$ over time intervals $[(k-1) \Lambda, k \Lambda)$ of (fixed) length $\Lambda$, for $k \in \mathbb{N}^{+}$, forms an i.i.d. sequence $\left\{\lambda_{k}\right\}_{k \geq 0}$. This model is generalized by Björk and Grandell (1988), who consider the intensity as $\lambda(t)=L_{i}$ if $\Sigma_{i-1} \leq t<\Sigma_{i}$, where $\Sigma_{i}=\sigma_{1}+\cdots+\sigma_{i}$, with $\Sigma_{0}=0$ and $\left(L_{i}, \sigma_{i}\right)$ a sequence of i.i.d. random vectors. Ammeter's model is revisited by Grandell (1995) and more properties of the model are discovered. 
Asmussen (1989) proposes a Cox risk model, called a Markov-modulated Poisson process, whose intensity process $\{\lambda(t)\}_{t \geq 0}$ is given by $\lambda(t)=\lambda_{J(t)}$. Here the process $\{J(t)\}_{t \geq 0}$ models the random environment of an insurance business and is assumed to be an irreducible continuous time Markov chain, with finite state space $\{1,2, \ldots, l\}$. Furthermore, a Cox risk process with a piecewise constant intensity is considered by Schmidli (1996), where the sequence $\left\{L_{i}\right\}_{i \geq 1}$ of successive levels of the intensity forms a Markov chain.

Ruin probabilities have been studied in these Cox models with a piecewise constant intensity. Lundberg inequalities hold, provided some assumptions are fulfilled. These may not be practical due to the difficulty in estimating the Lundberg coefficient and evaluating some constants within the inequalities. Other papers regarding to this topic are Embrechts et al. (1993) and Schmidli (1997).

There are very few results in the risk theory literature regarding Cox processes with other than piecewise constant intensities. Recently, Schmidli (2003) considered a NHP model with doubly stochastic occurrences for the PCS catastrophes index, based on individual indices for PCS options, where the intensity is of the form $\Lambda \lambda(t)$, with $\Lambda$ is stochastic and $\lambda(t)$ is a given function.

Some natural phenomena evolve in a seasonal environment subject to random fluctuations which, in turn, affect insurance claims. For example, tropical storms and hurricanes periodically affect the coastal US states along the Atlantic and the Gulf of Mexico. The claim intensity then forms a specific pattern for each year which can be modeled by a periodic function. Speculation exists regarding the significance and potential effects of the El Niño phenomenon on hurricane frequency and the strength attained by tropical cyclones during alternating El Niño/La Niña years. These are random effects that, in some sense, affect the risk propensity or the peak level of the seasonal intensity, which can be modeled by a stochastic process.

In this paper we propose a Cox model that accounts for both, the seasonal variations and the random fluctuations in the claims intensity. Beard et al. (1984) and Daykin et al. (1994) suggest an intensity process $\lambda$ as a composition of some factors, such as the normal trend, deviations from it and the short-term variations in risk propensity. Here we simply consider an intensity process with the following structure

$$
\lambda(t)=\lambda_{S}(t) \mathbf{q}(t), \quad t \geq 0
$$

where $\lambda_{S}(t)$ is the short-term intensity function and $\mathbf{q}(t)$ is a stochastic (level) process. The periodicity of the short-term intensity function is also considered, 
which takes into account those insurance claims affected by a periodic environment, like hurricanes or seasonal storms. A Markov chain with two states, corresponding to two different (high and low) levels, is chosen for the level process, yielding a so called regime-switching process. Under this intensity process, properties of the claim counting process and its corresponding risk process are studied in detail. By properly choosing the Lundberg coefficient, Lundberg-type upper bounds for finite time ruin probabilities are derived.

The paper is organized as follows. The model is defined in Section 2. Section 3 discusses the properties of the claim counting process. This gives a precise description of the model characteristics, such as the probabilities of recording $k$ claims during the time interval $[0, t)$, for $t \geq 0$ and $k \in \mathbb{N}$, and the expectation of the integrated intensities in (2). In Section 4 we derive Lundberg-type upper bounds for finite time ruin probabilities and illustrate the results by some examples.

\section{A Cox model with a regime-switching periodic intensity}

Consider an intensity process $\{\lambda(t)\}_{t \geq 0}$ governed by a deterministic pattern in each short-term period, say a year, and a random effect on its peak level, that is the amplitude of the pattern. This fixed intensity pattern can be seen as the short-term periodicity, like in the NHP process. Assume we have two different risk levels; $\lambda_{0}$ which represents the risk under "low season" conditions, while the other, $\lambda_{1}$, represents the peak intensity under "high risk" years. In practice, such conditions can be slippery roads, foggy days, stormy weather, years affected by the El Niño phenomenon and so on.

Furthermore, assume that the intensity level modulates by an irreducible discrete time Markov process, $\kappa=\left\{\kappa_{n}\right\}_{n \geq 0}$, with finite state space $\{0,1\}$ and the transition probability matrix $P$, given by

$$
P=\left(\begin{array}{cc}
1-p_{01} & p_{01} \\
p_{10} & 1-p_{10}
\end{array}\right) .
$$

Without loss of generality, we assume that the short-term period is 1 . Let $\beta$ be a function defined on $[0,1]$, such that $\beta\left(t^{*}\right)=1$, where $t^{*} \in[0,1]$ is the mode of the function. Consider the intensity process $\lambda$, given by

$$
\lambda(t)=\lambda_{\kappa_{\lfloor t\rfloor}} \beta(t-\lfloor t\rfloor), \quad t \geq 0 .
$$


This gives $\lambda\left(n+t^{*}\right)=\lambda_{\kappa_{n}} \beta\left(t^{*}\right)=\lambda_{\kappa_{n}}$ for $n \in \mathbb{N}$, that is, the peak of the function $\lambda(t)$ within the $(n+1)$-th year [i.e. $t \in[n, n+1)]$ is $\lambda_{\kappa_{n}}$, which changes according to the Markov chain $\kappa$. As such, we call $\lambda_{\kappa_{n}}$ the intensity level for year $n+1$.

In the sequel, we illustrate the annual common intensity pattern as a beta-type function with parameters $p \geq 1$ and $q \geq 1$, given by

$$
\beta(t)=\alpha^{*} t^{p-1}(1-t)^{q-1}, \quad 0 \leq t \leq 1,
$$

where $\alpha^{*}$ is a scale factor, given by

$$
\alpha^{*}=\frac{1}{\left(t^{*}\right)^{p-1}\left(1-t^{*}\right)^{q-1}} \quad \text { and } \quad t^{*}=\frac{p-1}{p+q-2}
$$

is the mode of $\beta(t), t \in[0,1]$. As such, note that at the mode $\beta\left(t^{*}\right)=1$ is the peak level [see Figure 1]. Also denote the beta function in the usual way

$$
B(p, q)=\int_{0}^{1} v^{p-1}(1-v)^{q-1} d v=\frac{\Gamma(p) \Gamma(q)}{\Gamma(p+q)}, \quad p, q \geq 1,
$$

and the incomplete beta function at $p, q \geq 1$ as

$$
B(p, q ; t)=\int_{0}^{t} v^{p-1}(1-v)^{q-1} d v, \quad t \in(0,1),
$$

with $B(p, q ; t)=0$ if $t \leq 0$, while $B(p, q ; t)=\lfloor t\rfloor B(p, q)+B(p, q ; t-\lfloor t\rfloor)$, if $t \geq 1$.
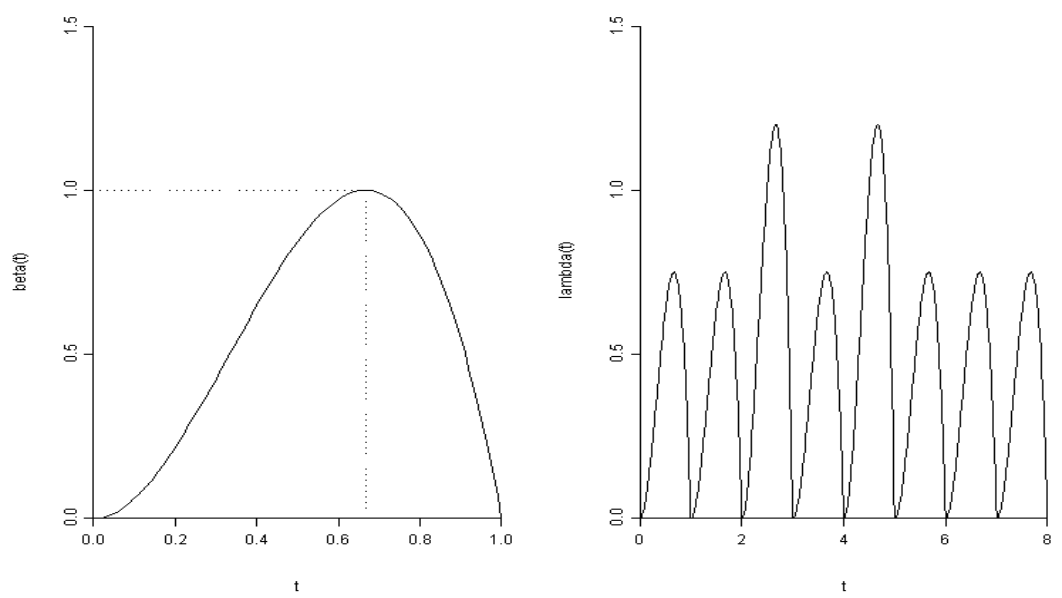

Figure 1: $\beta(t)$ and one realization of intensity process $\lambda$. 
Figure 1 illustrates function $\beta(t)$, when $p=3$ and $q=2$, as well as a realization of the intensity process $\lambda$, when $p=3, q=2, \lambda_{0}=0.75, \lambda_{1}=1.2, p_{01}=0.25$ and $p_{10}=0.5$.

Consider a special Cox process, the claim counting process $\{N(t)\}_{t \geq 0}$ with an intensity process as in (4). Due to the periodicity of the function $\beta(t-\lfloor t\rfloor)$, for $t \geq 0$, and the transitions, from year to year, between levels $\lambda_{0}$ and $\lambda_{1}$, we call this risk model a regime-switching periodic non-homogeneous Poisson (NHP) process.

Let $\left\{N_{i}(t)\right\}_{t \geq 0}$ for $i=0,1$, (with $N_{i}(0)=0$ ) denote a claim counting NHP process with intensity function $\lambda_{i} \beta(t-\lfloor t\rfloor)$ over the time interval $[0, t)$. That is $N_{i}(t)$ is Poisson distributed with mean $\lambda_{i} \int_{0}^{t} \beta(v-\lfloor v\rfloor) d v=\lambda_{i} \alpha^{*} B(p, q ; t)$. Then the process $\{N(t)\}_{t \geq 0}$ can be represented as

$$
N(t)=\sum_{i=0,1} Y_{i}(\lfloor t\rfloor) N_{i}(1)+N_{\kappa_{\lfloor t\rfloor}}(t)-N_{\kappa_{\lfloor t\rfloor}}(\lfloor t\rfloor), \quad t \geq 0
$$

where $Y_{i}(\lfloor t\rfloor)=\sum_{n=0}^{\lfloor t\rfloor-1} \mathbb{I}\left(\kappa_{n}=i\right)$ denotes the number of years in $[0,\lfloor t\rfloor)$ that $\kappa$ spends in state $i$, for $i=0,1$. This implies that, the conditional expected number of claims in the time interval $[0, t)$, given the environment, is:

$$
\begin{aligned}
& \mathbb{E}\left[N(t) \mid \kappa_{0}, \kappa_{1}, \ldots, \kappa_{\lfloor t\rfloor}\right] \\
& \quad=\sum_{n=0}^{\lfloor t\rfloor-1} \int_{0}^{1} \lambda_{\kappa_{n}} \beta(v) d v+\lambda_{\kappa_{\lfloor t\rfloor}} \int_{0}^{t-\lfloor t\rfloor} \beta(v) d v \\
& \quad=L(\lfloor t\rfloor) \alpha^{*} B(p, q)+\lambda_{\kappa_{\lfloor t\rfloor}} \alpha^{*} B(p, q ; t-\lfloor t\rfloor), \quad t \geq 0,
\end{aligned}
$$

where

$$
L(\lfloor t\rfloor)=Y_{0}(\lfloor t\rfloor) \lambda_{0}+Y_{1}(\lfloor t\rfloor) \lambda_{1}, \quad t \geq 0
$$

denotes the sum of $\lambda_{0}$ and $\lambda_{1}$ values in $[0,\lfloor t\rfloor)$. Hence, we have

$$
\mathbb{E}[N(t)] \leq \max \left\{\lambda_{0}, \lambda_{1}\right\} \alpha^{*} B(p, q ; t) .
$$

The corresponding compound NHP process $\{S(t)\}_{t \geq 0}$ is given by

$$
S(t)=\sum_{j=1}^{N(t)} X_{j}, \quad t \geq 0,
$$

where the $X_{j}$ 's are the claim sizes with distribution function $F_{X}$, expected claim size $\mu=\int_{0}^{\infty} v d F_{X}(v)$ and moment generating function $\hat{m}_{X}(s)=\int_{0}^{\infty} e^{s v} d F_{X}(v)$, 
for some $s>0$. These claim severities are assumed independent of the Markov environment process $\kappa$ and hence of the claim counting process $\{N(t)\}_{t \geq 0}$. As in (7) process $\{S(t)\}_{t \geq 0}$ can also be represented as

$$
S(t)=\sum_{i=0,1} Y_{i}(\lfloor t\rfloor) S_{i}(1)+S_{\kappa\lfloor t\rfloor}(t)-S_{\kappa\lfloor t\rfloor}(\lfloor t\rfloor), \quad t \geq 0
$$

where $S_{i}(t)=\sum_{j=1}^{N_{i}(t)} X_{j}$.

Now consider the continuous-time surplus process $\{U(t)\}_{t \geq 0}$, given by

$$
U(t)=u+c t-S(t), \quad t \geq 0,
$$

where $u$ is the initial capital value and $c$ is the constant premium rate. The aggregate claim process $\{S(t)\}_{t \geq 0}$ is given in (9) and the claim counting process $\{N(t)\}_{t \geq 0}$ is the regime-switching periodic NHP process in (7).

Since the Markov environment process $\kappa$ is assumed irreducible, it has a stationary initial distribution, denoted by $\pi=\left(\pi_{0}, \pi_{1}\right)$. Then by the law of large numbers for irreducible Markov processes, we have:

$$
\lim _{t \rightarrow \infty} \frac{U(t)}{t}=c-\mu \sum_{i=0,1} \pi_{i} \lambda_{i} \alpha^{*} B(p, q),
$$

[see Rolski et al. (1999, Chapter 12)].

(11) implies that ruin occurs almost surely if the process has a negative drift, that is $c \leq \mu \sum_{i=0,1} \pi_{i} \lambda_{i} \alpha^{*} B(p, q)$. Therefore we assume that the net profit condition

$$
c>\mu \sum_{i=0,1} \pi_{i} \lambda_{i} \alpha^{*} B(p, q)
$$

holds in the sequel.

\section{Properties of the regime--switching periodic process}

For the regime-switching periodic NHP process defined above, the random measure $\Lambda$ in this Cox process, given the realization of the environment process $\kappa$ up 
to time $\lfloor t\rfloor$, is:

$$
\Lambda(t)=\int_{0}^{t} \lambda(v) d v=L(\lfloor t\rfloor) \alpha^{*} B(p, q)+\lambda_{\kappa\lfloor t\rfloor} \alpha^{*} B(p, q ; t-\lfloor t\rfloor), \quad t \geq 0,
$$

where $L(\lfloor t\rfloor)$ is given in (8). Then the conditional probability that the number of claims is $k$ in the time interval $[0, t)$ is obtained as:

$$
P\left\{N(t)=k \mid \kappa_{0}, \kappa_{1}, \ldots, \kappa_{\lfloor t\rfloor}\right\}=\frac{[\Lambda(t)]^{k}}{k !} e^{-\Lambda(t)}, \quad k \in \mathbb{N}^{+},
$$

where $\Lambda(t)$ is given in (13).

In order to calculate $P\{N(t)=k\}$, we need to know how many times $\lambda_{0}$ appears in the sequence $\left\{\lambda_{\kappa_{0}}, \lambda_{\kappa_{1}}, \ldots, \lambda_{\kappa_{|t|}}\right\}$ (then the number of $\lambda_{1}$ values is fixed). This is equivalent to finding how many times 0 (say, "failure") or 1 ("success") appears in the corresponding sequence $\left\{\kappa_{0}, \kappa_{1}, \ldots, \kappa_{\lfloor t\rfloor}\right\}$. To do this, we denote $Y_{i}(n)$ to be the number of times that successive $n$-length sequences of the time-homogeneous $\{0,1\}$-valued Markov process $\kappa$ are in state $i$, for $i=0,1$.

Many papers discuss formulas or recursions for the distribution of success runs of several lengths in a two-state Markov chain [for example, see Han and Aki (1998)]. From these, it is not difficult to derive the distribution of the number of successes, $Y_{1}(n)$, which takes values in $\{0,1, \ldots, n\}$ and can be obtained as follows.

Let $E_{i}(n, y)$ denote the conditional probability of $y$ successes in a $(n+1)$-length sequence, given that the sequence starts from state $i$, for $i=0,1$. That is, $E_{0}(n, y)=P\left\{Y_{1}(n)=y\right\}$ and $E_{1}(n, y)=P\left\{Y_{1}(n)=y-1\right\}$. For convenience, define $E_{i}(n, y)=0$ for all $y<0, n \geq 0$ and $i=0,1$. We have the following recursive formulas for probabilities $E_{i}(n, y)$.

$$
\begin{aligned}
E_{i}(0,0)= & 1, \quad \text { for } i=0,1, \\
E_{0}(n, y)= & \left(1-p_{01}\right) E_{0}(n-1, y)+\sum_{m=1}^{n-1} p_{01}\left(1-p_{10}\right)^{m-1} p_{10} E_{0}(n-m-1, y-m) \\
& \quad+p_{01}\left(1-p_{10}\right)^{n-1} E_{1}(0, y-n), \quad \text { for } 0 \leq y \leq n, n \geq 1, \quad \text { (14) } \\
E_{1}(n, y)= & p_{10} E_{0}(n-1, y)+\sum_{m=1}^{n-1}\left(1-p_{10}\right)^{m} p_{10} E_{0}(n-m-1, y-m) \\
& +\left(1-p_{10}\right)^{n} E_{1}(0, y-n), \quad \text { for } 0 \leq y \leq n, n \geq 1 .
\end{aligned}
$$

Denote by $P_{n}(y)$, the probability of $Y_{1}(n)=y$ (implying that $Y_{0}(n)$ must be $n-y$ ) in a $n$-length sequence of the $\{0,1\}$-valued irreducible Markov chain $\kappa$. Then 
assuming that this $n$-length sequence starts $\kappa_{0}$, the law of the total probabilities gives:

$$
\begin{aligned}
P_{n}(y) & =\sum_{i=0,1} P\left\{Y_{1}(n)=y \mid \kappa_{0}=i\right\} P\left\{\kappa_{0}=i\right\} \\
& =\pi_{0} P\left\{Y_{1}(n-1)=y \mid \kappa_{0}=0\right\}+\pi_{1} P\left\{Y_{1}(n-1)=y-1 \mid \kappa_{0}=1\right\} \\
& =\pi_{0} E_{0}(n-1, y)+\pi_{1} E_{1}(n-1, y-1) \\
& =\sum_{i=0,1} \pi_{i} E_{i}(n-1, y-i), \quad \text { for } 0 \leq y \leq n, n \in \mathbb{N},
\end{aligned}
$$

where $E_{i}(n-1, y-i)$ can be recursively calculated from (14) and $\left(\pi_{0}, \pi_{1}\right)$ is the initial distribution of Markov chain $\kappa$.

For example, in a 3-length sequence, the probability that there are no successes is given by:

$P_{3}(0)=\pi_{0} E_{0}(2,0)=\pi_{0}\left(1-p_{01}\right) E_{0}(1,0)=\pi_{0}\left(1-p_{01}\right)^{2} E_{0}(0,0)=\pi_{0}\left(1-p_{01}\right)^{2}$, since first it has to start from state 0 and then stays at 0 (failure) for the next two steps. By contrast, the probability that there are 1 success and 2 failures is

$$
\begin{aligned}
P_{3}(1) & =\pi_{0} E_{0}(2,1)+\pi_{1} E_{1}(2,0) \\
& =\pi_{0}\left[\left(1-p_{01}\right) E_{0}(1,1)+p_{01} p_{10} E_{0}(0,0)\right]+\pi_{1} p_{10} E_{0}(1,0) \\
& =\pi_{0}\left[\left(1-p_{01}\right) p_{01} E_{1}(0,0)+p_{01} p_{10}\right]+\pi_{1} p_{10}\left(1-p_{01}\right) E_{0}(0,0) \\
& =\pi_{0}\left[\left(1-p_{01}\right) p_{01}+p_{01} p_{10}\right]+\pi_{1} p_{10}\left(1-p_{01}\right)
\end{aligned}
$$

since if the sequence starts at 0 , it must go to 1 once in the next two steps. But if the sequence starts at 1 , it has to stay at 0 for the next two steps, and so on. Similarly, we have $P_{3}(2)=\pi_{0} p_{01}\left(1-p_{10}\right)+\pi_{1} p_{10}\left[p_{01}+\left(1-p_{10}\right)\right]$ and $P_{3}(3)=\pi_{1}\left(1-p_{10}\right)^{2}$.

We introduce the following notation for abbreviation. Denote by $\Lambda_{n}(y)$ the random measure under a realization of $y$ periods at level $\lambda_{1}$ (and hence $n-y$ periods at $\left.\lambda_{0}\right)$, in the sequence $\left\{\lambda_{\kappa_{0}}, \lambda_{\kappa_{1}}, \ldots, \lambda_{\kappa_{n-1}}\right\}$. That is

$$
\Lambda_{n}(y)=\left[(n-y) \lambda_{0}+y \lambda_{1}\right] \alpha^{*} B(p, q), \quad 0 \leq y \leq n, n \in \mathbb{N} .
$$

Then we have the following theorem for the probabilities $P\{N(t)=k\}$.

Theorem 1 Let $\kappa=\left\{\kappa_{n}\right\}_{n \geq 0}$ be a $\{0,1\}$-valued irreducible Markov chain with transition probabilities given by $(3)$ and initial distribution $\left(\pi_{0}, \pi_{1}\right)$. For the coun- 
ting process $\{N(t)\}_{t \geq 0}$, given by $(7)$, the probabilities that there be $k$ claim occurrences during the time interval $[0, t)$, for $t \geq 0$ and $k \in \mathbb{N}$, is given by

$$
\begin{aligned}
P\{N(t)=k\}=\sum_{y=0}^{\lfloor t\rfloor} P_{\lfloor t\rfloor}(y)\{ & \sum_{i=0,1}\left(\pi_{0} p_{0 i}+\pi_{1} p_{1 i}\right) e^{-\left[\Lambda_{\lfloor t\rfloor}(y)+\lambda_{i} \alpha^{*} B(p, q ; t-\lfloor t\rfloor)\right]} \\
& \left.\frac{\left[\Lambda_{\lfloor t\rfloor}(y)+\lambda_{i} \alpha^{*} B(p, q ; t-\lfloor t\rfloor)\right]^{k}}{k !}\right\}
\end{aligned}
$$

where $P_{\lfloor t\rfloor}(y)$ and $\Lambda_{\lfloor t\rfloor}(y)$ are obtained from (15) and (16), respectively.

Proof. See the Appendix.

Note here that (17) can be re-written as

$$
P\{N(t)=k\}=\mathbb{E}\left[P\left\{N(t)=k \mid \kappa_{0}, \kappa_{1}, \ldots, \kappa_{\lfloor t\rfloor}\right\}\right]=\mathbb{E}\left[\frac{\Lambda(t)^{k}}{k !} e^{-\Lambda(t)}\right],
$$

where $\Lambda(t)$ is given by (13). It means that this regime-switching periodic NHP process can also be interpreted as a mixed Poisson process.

The random measure $\Lambda(t)$ of this special Cox process is given by (13). Taking expectations in (13) directly gives

$$
\begin{aligned}
\mathbb{E}[\Lambda(t)] & =\mathbb{E}\left[L(\lfloor t\rfloor) \alpha^{*} B(p, q)+\lambda_{\kappa_{\lfloor t\rfloor}} \alpha^{*} B(p, q ; t-\lfloor t\rfloor)\right] \\
& =\alpha^{*} B(p, q) \mathbb{E}[L(\lfloor t\rfloor)]+\alpha^{*} B(p, q ; t-\lfloor t\rfloor) \mathbb{E}\left[\lambda_{\kappa_{\lfloor t\rfloor}}\right],
\end{aligned}
$$

then since $\mathbb{E}\left[Y_{1}(\lfloor t\rfloor)\right]=\sum_{y=0}^{\lfloor t\rfloor} y P_{\lfloor t\rfloor}(y)$ and $\mathbb{E}\left[\lambda_{\kappa_{\lfloor t\rfloor}}\right]=\lambda_{0}\left[\pi_{0}\left(1-p_{01}\right)+\pi_{1} p_{10}\right]+$ $\lambda_{1}\left[\pi_{0} p_{01}+\pi_{1}\left(1-p_{10}\right)\right]$, it follows that

$$
\begin{aligned}
\mathbb{E}[\Lambda(t)]=\alpha^{*} B(p, q) \sum_{y=0}^{\lfloor t\rfloor} P_{\lfloor t\rfloor}(y)\left[(\lfloor t\rfloor-y) \lambda_{0}+y \lambda_{1}\right] \\
\quad+\alpha^{*} B(p, q ; t-\lfloor t\rfloor) \sum_{i=0,1} \lambda_{i}\left(\pi_{0} p_{0 i}+\pi_{1} p_{1 i}\right), \quad t \geq 0 .
\end{aligned}
$$

It is not difficult to see that (18) is equivalent to

$$
\mathbb{E}[\Lambda(t)]=\sum_{y=0}^{\lfloor t\rfloor} P_{\lfloor t\rfloor}(y) \sum_{i=0,1}\left(\pi_{0} p_{0 i}+\pi_{1} p_{1 i}\right)\left[\Lambda_{\lfloor t\rfloor}(y)+\lambda_{i} \alpha^{*} B(p, q ; t-\lfloor t\rfloor)\right],
$$


then when $t \geq 0$ and $s<a_{\Lambda}$, the moment generating function of $\Lambda(t), \hat{m}_{\Lambda(t)}(s)=$ $\mathbb{E}[\exp \{s \Lambda(t)\}]$, is given by

$$
\hat{m}_{\Lambda(t)}(s)=\sum_{y=0}^{\lfloor t\rfloor} P_{\lfloor t\rfloor}(y) \sum_{i=0,1}\left(\pi_{0} p_{0 i}+\pi_{1} p_{1 i}\right) e^{s\left[\Lambda_{\lfloor t\rfloor}(y)+\lambda_{i} \alpha^{*} B(p, q ; t-\lfloor t\rfloor)\right]},
$$

where $a_{\Lambda} \in \mathbb{R}^{+}$is such that $\lim _{s \uparrow a_{\Lambda}} \hat{m}_{\Lambda(t)}(s)=+\infty$, while $P_{\lfloor t\rfloor}(y)$ can be obtained from (15).

It is interesting to see that (19) can be re-written as

$$
\begin{aligned}
\hat{m}_{\Lambda(t)}(s) & =\sum_{y=0}^{\lfloor t\rfloor} P_{\lfloor t\rfloor}(y) e^{s \Lambda_{\lfloor t\rfloor}(y)} \sum_{i=0,1}\left(\pi_{0} p_{0 i}+\pi_{1} p_{1 i}\right) e^{s \lambda_{i} \alpha^{*} B(p, q ; t-\lfloor t\rfloor)} \\
& =\hat{m}_{\Lambda(\lfloor t\rfloor)}(s) \hat{m}_{\Lambda(t-\lfloor t\rfloor)}(s), \quad s<a_{\Lambda},
\end{aligned}
$$

showing that $\Lambda(t)=\Lambda(\lfloor t\rfloor)+\Lambda(t-\lfloor t\rfloor)$ and that these are independent.

Theorem 1 and the above results on $\Lambda(t)$ allow for the derivation of the moments of $N(t)$. For instance, applying Fubini's Theorem and simple manipulations to (17), gives the probability generating function $\hat{g}_{N(t)}(s)=\mathbb{E}\left[s^{N(t)}\right]$ :

$$
\begin{aligned}
\hat{g}_{N(t)}(s) & =\sum_{y=0}^{\lfloor t\rfloor} P_{\lfloor t\rfloor}(y) \sum_{i=0,1}\left(\pi_{0} p_{0 i}+\pi_{1} p_{1 i}\right) e^{(s-1)\left[\Lambda_{\lfloor t\rfloor}(y)+\lambda_{i} \alpha^{*} B(p, q ; t-\lfloor t\rfloor)\right]} \\
& =\mathbb{E}\left[e^{(s-1) \Lambda(t)}\right]=\hat{m}_{\Lambda}(s-1), \quad|s|<1 .
\end{aligned}
$$

Furthermore, taking the $r$-th derivative of $\hat{g}_{N(t)}(s)$ with respect to $s \in(0,1)$, $\hat{g}_{N(t)}^{(r)}(s)$, and its limit as $s \uparrow 1$, yields the following successive factorial moments of $N(t)$ (that these be finite or not):

$$
\begin{aligned}
\mathbb{E}[N(t)[N(t)-1] \cdots[N(t)-r+1]] & =\hat{g}_{N(t)}^{(r)}(1)=\lim _{s \uparrow 1} \hat{g}_{N(t)}^{(r)}(s) \\
& =\lim _{s \uparrow 1} \mathbb{E}\left[\Lambda(t)^{r} e^{(s-1) \Lambda(t)}\right]=\mathbb{E}\left[\Lambda(t)^{r}\right] .
\end{aligned}
$$

In particular, we have that:

$$
\mathbb{E}[N(t)]=\mathbb{E}[\Lambda(t)] \quad \text { and } \quad \mathbb{V}[N(t)]=\mathbb{V}[\Lambda(t)]+\mathbb{E}[\Lambda(t)]
$$

which imply that the index of dispersion of $N(t)$ is $\mathbb{I}_{N(t)}=\frac{\mathbb{V}[N(t)]}{\mathbb{E}[N(t)]}=1+\mathbb{I}_{\Lambda(t)}>1$, showing that $N(t)$ is overdispersed, by contrast to the classical Poisson process. 


\section{A Lundberg upper bound for finite time ruin probabilities}

This last section discusses the ruin problem for our special Cox process. The risk (income) process, over the time interval $[0, t)$, with initial value $R(0)=0$ and a constant premium rate $c$, is given as

$$
R(t)=c t-S(t)=c t-\sum_{j=1}^{N(t)} X_{j}, \quad t \geq 0
$$

where the claim counting process $\{N(t)\}_{t \geq 0}$ is the regime-switching periodic NHP process driven by our $\{0,1\}$-valued Markov chain $\kappa$ and $S(t)$ is as in (9). Further assume that the moment generating function $\hat{m}_{X}(s)=\int_{0}^{\infty} e^{s x} d F_{X}(x)$ is twice differentiable on an interval $\left[0, a_{X}\right)$, where $a_{X}>0$ and $\lim _{s \uparrow a_{X}} \hat{m}_{X}(s)=+\infty$.

Denote the Laplace-Stieltjes transform of $R(t)$ by $l(r ; t)=\mathbb{E}\left[e^{-r R(t)}\right]$. Assuming it exists, it is given by

$$
l(r ; t)=e^{\Lambda(t)\left[\hat{m}_{X}(r)-1\right]-r c t}, \quad r>a_{R(t)}, t \geq 0 .
$$

Similarly, for $i=0,1$, let

$$
\begin{aligned}
& l_{i}(r ; t)=\mathbb{E}\left[e^{-r R_{i}(t)}\right]=\mathbb{E}\left[e^{-r\left(c t-\sum_{j=1}^{N_{i}(t)} X_{j}\right)}\right] \\
& =e^{\lambda_{i} \alpha^{*} B(p, q ; t)\left[\hat{m}_{X}(r)-1\right]-r c t}, \quad r>a_{R_{i}(t)}, t \geq 0 .
\end{aligned}
$$

Let the time to ruin be defined in the usual way:

$$
T_{u}=\inf \{t \geq 0 \mid u+R(t)<0\}, \quad u \geq 0 .
$$

The ultimate ruin probability $\Psi(u)$ is then given by:

$$
\Psi(u)=P\left\{T_{u}<\infty\right\}, \quad u \geq 0 .
$$

Using the martingale approach to Cox models discussed in Grandell (1991) we can prove the following result.

Theorem 2 The following Lundberg-type upper bound holds for the finite time ruin probability in model (21):

$$
P\left\{T_{u} \leq t_{0}\right\} \leq e^{-r u} \mathbb{E}\left[\sup _{0 \leq t \leq t_{o}} l(r ; t)\right], \quad 0 \leq t_{0}<\infty
$$


A tighter upper bound can also be obtained for $0 \leq t_{0}<\infty$, as:

$$
P\left\{T_{u} \leq t_{0}\right\} \leq e^{-r u} \mathbb{E}\left[\sup _{0 \leq t \leq t_{o}} l(r ; t)\right] \sup _{y \geq 0}\left\{\frac{e^{r y} \bar{F}_{X}(y)}{\int_{y}^{\infty} e^{r x} d F_{X}(x)}\right\},
$$

where $\bar{F}_{X}=1-F_{X}$ is the tail of the distribution function of $X$.

Proof. For details see the Appendix.

The upper bound given in (25) is difficult to use in practice. To derive a corresponding useful bound for our regime-switching periodic NHP model, first define the average risk level, given by

$$
\bar{\lambda}=\pi_{0} \lambda_{0}+\pi_{1} \lambda_{1}
$$

and consider, for $r \geq 0$, the equation

$$
\theta(r)=\bar{\lambda} \alpha^{*} B(p, q)\left[\hat{m}_{X}(r)-1\right]-r c=0 .
$$

The solution, $\gamma>0$, to (27) satisfies:

$$
\bar{\lambda} \alpha^{*} B(p, q)\left[\hat{m}_{X}(\gamma)-1\right]=\gamma c .
$$

Here $\gamma$ is an adjustment coefficient for the average risk level $\bar{\lambda}$ in (26), where $\lambda_{1}$, the peak intensity under "high risk" years, is assumed larger than that in the "low season" (i.e. $\lambda_{0}<\lambda_{1}$ ). It follows from (28) that

$$
\lambda_{i} \alpha^{*} B(p, q)\left[\hat{m}_{X}(\gamma)-1\right]=\frac{\lambda_{i}}{\bar{\lambda}} \gamma c, \quad i=0,1 .
$$

The existence and unicity of $\gamma$ in $\left[0, a_{X}\right)$ is guaranteed because $\theta(0)=0$ and $\theta^{\prime}(0)=\bar{\lambda} \alpha^{*} B(p, q) \mu-c<0$, provided that the net profit condition (12) holds, and hence the convexity of $\theta(r)$ ensures that $\theta^{\prime}(\gamma)>0$.

Assume that $t_{0}$ is an integer. Then with probabilities $P_{t_{0}}(y)$, given by $(15), \Lambda\left(t_{0}\right)$ takes the following realizations:

$$
\Lambda_{t_{0}}(y)=\left[\left(t_{0}-y\right) \lambda_{0}+y \lambda_{1}\right] \alpha^{*} B(p, q), \quad 0 \leq y \leq t_{0}, t_{0} \in \mathbb{N} .
$$

When $0 \leq t \leq t_{0}$, we have two possibilities for $\Lambda(t)$, depending on the value of $\lambda_{\kappa_{\lfloor t\rfloor}}$. One is

$$
\Lambda(t)=\left[(\lfloor t\rfloor-z) \lambda_{0}+z \lambda_{1}\right] \alpha^{*} B(p, q)+\lambda_{0} \alpha^{*} B(p, q ; t-\lfloor t\rfloor), \quad 0 \leq t \leq t_{0},
$$


where $0 \leq z \leq \min \{\lfloor t\rfloor, y\}$ and $\lfloor t\rfloor-z+1 \leq t_{0}-y$, or equivalently, $z \in C(t+1, y)=$ $\left[\max \left\{0,\lfloor t\rfloor+1-\left(t_{0}-y\right)\right\}, \min \{\lfloor t\rfloor, y\}\right]$. While the other is

$$
\Lambda(t)=\left[(\lfloor t\rfloor-z) \lambda_{0}+z \lambda_{1}\right] \alpha^{*} B(p, q)+\lambda_{1} \alpha^{*} B(p, q ; t-\lfloor t\rfloor), \quad 0 \leq t \leq t_{0},
$$

where similarly, $0 \leq z \leq \min \{\lfloor t\rfloor, y-1\}$ and $\lfloor t\rfloor-z \leq t_{0}-y$, or equivalently, $z \in C(t, y-1)=\left[\max \left\{0,\lfloor t\rfloor-\left(t_{0}-y\right)\right\}, \min \{\lfloor t\rfloor, y-1\}\right]$.

When $\Lambda(t)$ is given by (30), then (28) and (29) imply that:

$$
\begin{aligned}
\Lambda(t)\left[\hat{m}_{X}\right. & (\gamma)-1]-\gamma c t=(\lfloor t\rfloor-z)\left[\lambda_{0} \alpha^{*} B(p, q)\left(\hat{m}_{X}(\gamma)-1\right)-\gamma c\right] \\
& +z\left[\lambda_{1} \alpha^{*} B(p, q)\left(\hat{m}_{X}(\gamma)-1\right)-\gamma c\right] \\
& +\lambda_{0} \alpha^{*} B(p, q ; t-\lfloor t\rfloor)\left(\hat{m}_{X}(\gamma)-1\right)-\gamma c(t-\lfloor t\rfloor) \\
=- & \lfloor t\rfloor\left(\frac{\bar{\lambda}-\lambda_{0}}{\bar{\lambda}}\right) \gamma c+z\left(\frac{\lambda_{1}-\lambda_{0}}{\bar{\lambda}}\right) \gamma c \\
& +\lambda_{0} \alpha^{*} B(p, q ; t-\lfloor t\rfloor)\left[\hat{m}_{X}(\gamma)-1\right]-\gamma c(t-\lfloor t\rfloor) .
\end{aligned}
$$

In turn

$$
\begin{aligned}
\sup _{0 \leq t \leq t_{0}} l(\gamma ; t) & =\sup _{0 \leq t \leq t_{0}} e^{\Lambda(t)\left[\hat{m}_{X}(\gamma)-1\right]-\gamma c t} \\
& \leq \sup _{\substack{0 \leq t \leq t_{0} \\
z \in C(t+1, y)}} e^{z\left(\frac{\lambda_{1}-\lambda_{0}}{\lambda}\right) \gamma c+\lambda_{0} \alpha^{*} B(p, q ; t-\lfloor t])\left[\hat{m}_{X}(\gamma)-1\right]-\gamma c(t-\lfloor t])} \\
& =\max _{\substack{0 \leq t \leq t_{0} \\
z \in C(t+1, y)}} e^{z\left(\frac{\lambda_{1}-\lambda_{0}}{\lambda}\right) \gamma c} \max _{0 \leq v<1} l_{0}(\gamma ; v) \\
& \leq e^{y\left(\frac{\lambda_{1}-\lambda_{0}}{\lambda}\right) \gamma c} \max _{0 \leq v<1} l_{0}(\gamma ; v)
\end{aligned}
$$

Similarly, when $\Lambda(t)$ is given by (31), then

$$
\sup _{0 \leq t \leq t_{0}} l(\gamma ; t) \leq e^{(y-1)\left(\frac{\lambda_{1}-\lambda_{0}}{\lambda}\right) \gamma c} \max _{0 \leq v<1} l_{1}(\gamma ; v) \leq e^{y\left(\frac{\lambda_{1}-\lambda_{0}}{\lambda}\right) \gamma c} \max _{0 \leq v<1} l_{1}(\gamma ; v),
$$

which has the same form as (32). Taking expectations gives

$$
\mathbb{E}\left[\sup _{0 \leq t \leq t_{o}} l(r ; t)\right]=\sum_{y=0}^{t_{0}} P_{t_{0}}(y) e^{y\left(\frac{\lambda_{1}-\lambda_{0}}{\lambda}\right) \gamma c} .
$$


Finally, setting $r=\gamma$, gives a Lundberg-type upper bound for the finite time ruin probability in $(25)$, for $t_{0} \in \mathbb{N}$, that is:

$P\left\{T_{u} \leq t_{0}\right\} \leq e^{-\gamma u}\left[\sum_{y=0}^{t_{0}} P_{t_{0}}(y) e^{y\left(\frac{\lambda_{1}-\lambda_{0}}{\lambda}\right) \gamma c}\right] \max _{\substack{0 \leq v<1 \\ i=0,1}} l_{i}(\gamma ; v) \sup _{y \geq 0}\left\{\frac{e^{r y} \bar{F}_{X}(y)}{\int_{y}^{\infty} e^{r x} d F_{X}(x)}\right\}$,

where $\gamma$ satisfies $(28)$ and $P_{t_{0}}(y)$ is given in (15).

Obviously, the simpler bound for $P\left\{T_{u} \leq t_{0}\right\}$ given by (24) can also be derived here:

$$
P\left\{T_{u} \leq t_{0}\right\} \leq e^{-\gamma u}\left[\sum_{y=0}^{t_{0}} P_{t_{0}}(y) e^{y\left(\frac{\lambda_{1}-\lambda_{0}}{\lambda}\right) \gamma c}\right] \max _{\substack{0 \leq v<1 \\ i=0,1}} l_{i}(\gamma ; v)
$$

but (33) is tighter than (34), as shown in the following examples.

Example 1 Consider claim sizes that are exponentially distributed with mean $\mu$. Their moment generating function $\hat{m}_{X}(s)=\frac{1}{1-\mu s}$, for $s<a_{X}=\frac{1}{\mu}$. The adjustment coefficient for parameter $\lambda_{0}$, is then given by

$$
\gamma=\frac{c-\lambda_{0} \alpha^{*} B(p, q) \mu}{c \mu}=\frac{1}{\mu}-\frac{\bar{\lambda} \alpha^{*} B(p, q)}{c}
$$

which is the positive solution to equation (28). The corresponding $l_{i}(\gamma ; v)$, given in (23), takes the form

$$
l_{i}(\gamma ; v)=e^{\left(\frac{\lambda_{i}}{\lambda} \frac{B(p, q ; v)}{B(p, q)}-v\right) \gamma c}, \quad 0 \leq v<1, i=0,1
$$

Figure 2 illustrates the upper bounds in this exponential case, as a function of $u$ (left graph), when $t_{0}=20$, and as a function of $t_{0}$ (right graph), when $u=10$. The other parameters are chosen to be $\lambda_{0}=1, \lambda_{1}=1.2, p=3, q=2, p_{01}=0.25$, $p_{10}=0.5, c=1.5, \mu=1.5$ and $\gamma=0.267$, which is obtained from (35). Clearly, the upper bounds $(a)$, given by (33) are sharper than those in (b), given by (34).

Example 2 Consider the case of inverse Gaussian distributed claims, with mean $\mu$, variance $\mu \beta$ and density function

$$
f_{X}(x)=\frac{\mu}{\sqrt{2 \pi \beta x^{3}}} e^{-\frac{(x-\mu)^{2}}{2 \beta x}}, \quad x>0 .
$$



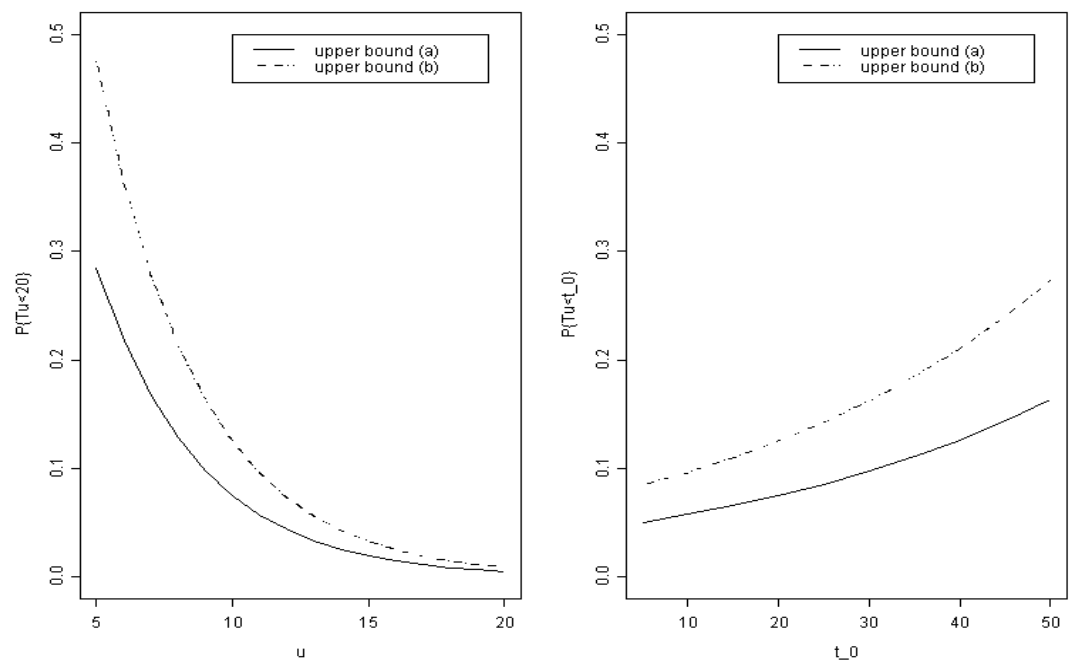

Figure 2: Upper bounds for exponential claims vs $u\left(t_{0}=20\right)$ and $t_{0}(u=10)$.

Their moment generating function $\hat{m}_{X}(s)=e^{\frac{\mu}{\beta}(1-\sqrt{1-2 \beta s})}$ exists for $s<\frac{1}{2 \beta}$. The adjustment coefficient $\gamma$ with respect to parameter $\bar{\lambda}$ is the positive solution to the equation

$$
\bar{\lambda} \alpha^{*} B(p, q)\left[e^{\frac{\mu}{\beta}(1-\sqrt{1-2 \beta \gamma})}-1\right]=\gamma c
$$

and $l_{i}(v ; \gamma)$, for $i=0,1$, is of the same form as in (36).

Figure 3 illustrates the upper bounds in this inverse Gaussian case, again as a function of $u$ (left graph), when $t_{0}=20$, and as a function of $t_{0}$ (right graph), when $u=10$. The other parameters are chosen as for Figure 2 and $\beta=\frac{8}{3}$, which gives a variance of 4 . Here $\gamma=0.155$ is obtained from (37). Again the upper bounds in $(a)$, given by (33) are sharper than those in (b), given by (34).

\section{Conclusions}

Regime-switching periodic NHP processes can be useful in modeling risk processes under periodic and random environments. A beta-type short-term intensity function is proposed with a two-state Markov process to model the peak level in the intensity of this Cox risk process. This generalizes the periodic NHP model. It can 

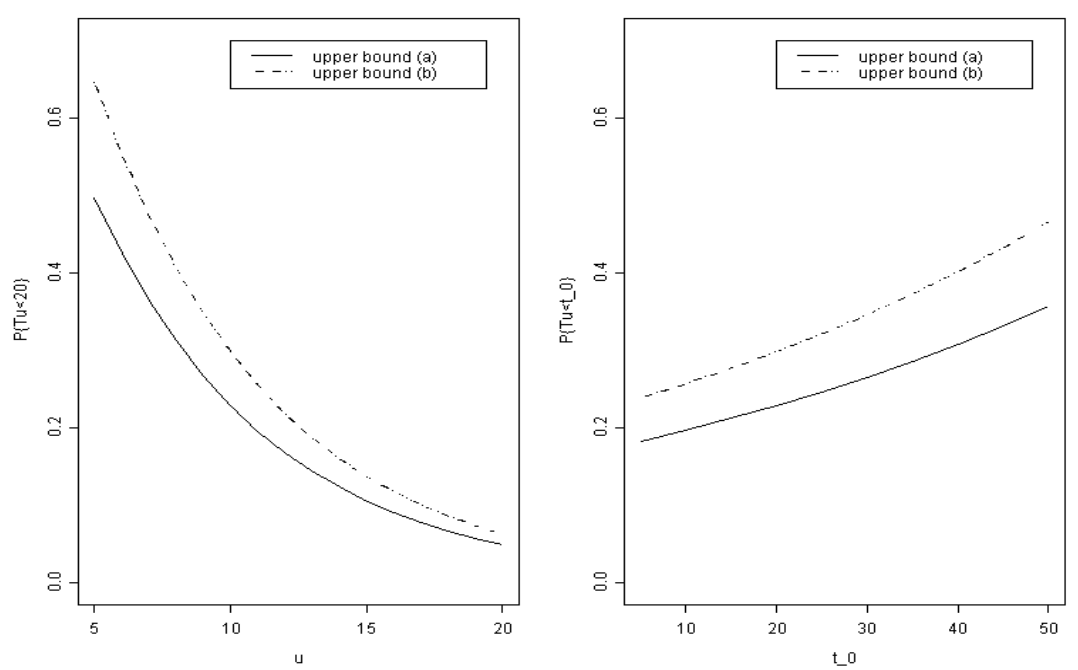

Figure 3: Upper bounds for inverse Gaussian claims vs $t_{0}(u=10)$ and $u\left(t_{0}=20\right)$.

also provide more realistic descriptions than Cox models with piecewise constant intensities.

The flexible shape of the beta function and the explicit results obtained for the Cox risk process should make these regime-switching period NHP models more practical than Cox processes with piecewise constant intensities, or than the usual NHP process. However, this work can be extended to other reasonable short-term intensity functions or regime-switching level processes with multi-state spaces. Furthermore, statistical methods to estimate from real data set the beta parameters and level parameters of the model are readily available and shall be illustrated in subsequent work.

\section{Appendix}

Proof of Theorem 1: By the law of the total probabilities, it is easily seen that

$$
\begin{aligned}
& P\{N(t)=k\}=P\{N(\lfloor t\rfloor)+[N(t)-N(\lfloor t\rfloor)]=k\} \\
& \quad=\sum_{l=0}^{k} P\{N(\lfloor t\rfloor)=l\} P\{N(t)-N(\lfloor t\rfloor)=k-l\} .
\end{aligned}
$$


Furthermore, since

$$
\begin{aligned}
P\{N(\lfloor t\rfloor)=l\} & \left.=\sum_{y=0}^{\lfloor t\rfloor} P\left\{N(\lfloor t\rfloor)=l \mid Y_{1}\lfloor t\rfloor\right)=y\right\} P\left\{Y_{1}(\lfloor t\rfloor)=y\right\} \\
& =\sum_{y=0}^{\lfloor t\rfloor} \frac{\left[\Lambda_{\lfloor t\rfloor}(y)\right]^{l}}{l !} e^{-\Lambda_{\lfloor t\rfloor}(y)} P_{\lfloor t\rfloor}(y)
\end{aligned}
$$

and

$$
\begin{aligned}
& P\{N(t)-N(\lfloor t\rfloor)=k-l\} \\
& =\sum_{i=0,1} P\left\{N(t)-N(\lfloor t\rfloor)=k-l \mid \kappa_{\lfloor t\rfloor-1}=i\right\} P\left\{\kappa_{\lfloor t\rfloor-1}=i\right\} \\
& =\sum_{i=0,1}\left[\sum_{j=0,1} P\left\{N(t)-N(\lfloor t\rfloor)=k-l \mid \kappa_{\lfloor t\rfloor-1}=i, \kappa_{\lfloor t\rfloor}=j\right\}\right. \\
& \left.P\left\{\kappa_{\lfloor t\rfloor}=j \mid \kappa_{\lfloor t\rfloor-1}=i\right\}\right] \pi_{i} \\
& =\sum_{i=0,1}\left(\pi_{0} p_{0 i}+\pi_{1} p_{1 i}\right) \frac{\left[\lambda_{i} \alpha^{*} B(p, q ; t-\lfloor t\rfloor)\right]^{k-l}}{(k-l) !} e^{-\lambda_{i} \alpha^{*} B(p, q ; t-\lfloor t\rfloor)},
\end{aligned}
$$

we now can write

$$
\begin{gathered}
P\{N(t)=k\}=\sum_{y=0}^{\lfloor t\rfloor} P_{\lfloor t\rfloor}(y)\left\{\sum_{i=0,1}\left(\pi_{0} p_{0 i}+\pi_{1} p_{1 i}\right) e^{-\left[\Lambda_{\lfloor t\rfloor}(y)+\lambda_{i} \alpha^{*} B(p, q ; t-\lfloor t\rfloor)\right]}\right. \\
\left.\sum_{l=0}^{k} \frac{\left[\Lambda_{\lfloor t\rfloor}(y)\right]^{l}}{l !(k-l) !}\left[\lambda_{i} \alpha^{*} B(p, q ; t-\lfloor t\rfloor)\right]^{k-l}\right\} \\
=\sum_{y=0}^{\lfloor t\rfloor} P_{\lfloor t\rfloor}(y)\left\{\sum_{i=0,1}\left(\pi_{0} p_{0 i}+\pi_{1} p_{1 i}\right) e^{-\left[\Lambda_{\lfloor t\rfloor}(y)+\lambda_{i} \alpha^{*} B(p, q ; t-\lfloor t\rfloor)\right]}\right. \\
\left.\frac{\left[\Lambda_{\lfloor t\rfloor}(y)+\lambda_{i} \alpha^{*} B(p, q ; t-\lfloor t\rfloor)\right]^{k}}{k !}\right\},
\end{gathered}
$$

which completes the proof.

Proof of Theorem 2: Consider the martingale approach to Cox models discussed in Grandell (1991). Let $\mathbf{F}$ be a suitable filtration, $M$ be a positive $\mathbf{F}$-martingale (or a positive $\mathbf{F}$-supermartingale) and $T$ be an $\mathbf{F}$-stopping time. Choose $t_{0}<\infty$ and consider $t_{0} \wedge T$, a bounded $\mathbf{F}$-stopping time. 
By the optional stopping theorem, we have that

$$
M(0) \geq \mathbb{E}^{\mathcal{F}_{0}}\left[M\left(t_{0} \wedge T\right)\right] \geq \mathbb{E}^{\mathcal{F}_{0}}\left[M(T) \mid t \leq t_{0}\right] P^{\mathcal{F}_{0}}\left\{T \leq t_{0}\right\},
$$

and therefore

$$
P^{\mathcal{F}_{0}}\left\{T \leq t_{0}\right\} \leq \frac{M(0)}{\mathbb{E}^{\mathcal{F}_{0}}\left[M(T) \mid T \leq t_{0}\right]}, \quad t_{0}<\infty
$$

Let the risk process $R$ be adapted to $\mathbf{F}$, that is $\mathcal{F}_{t} \supseteq \mathcal{F}_{t}^{R}$ for all $t \geq 0$. Then the ultimate ruin probability $\Psi(u)$ is seen to be:

$$
\Psi(u)=P\left\{T_{u}<\infty\right\}=\mathbb{E}\left[P^{\mathcal{F}_{0}}\left\{T_{u}<\infty\right\}\right], \quad u \geq 0 .
$$

Now consider $N$ to be a Cox process with intensity process $\{\lambda(t)\}_{t \geq 0}$ and random intensity measure $\Lambda$, given by $\Lambda(t)=\int_{0}^{t} \lambda(v) d v$. A suitable filtration $\mathbf{F}$ is defined as $\mathcal{F}_{t}=\mathcal{F}_{\infty}^{\Lambda} \vee \mathcal{F}_{t}^{R}$ and thus $\mathcal{F}_{0}=\mathcal{F}_{\infty}^{\Lambda}$. Consider the following choice of process $M$ :

$$
M(t)=\frac{e^{-r[u+R(t)]}}{l(r ; t)}=\frac{e^{-r[u+R(t)]}}{e^{\Lambda(t)\left[\hat{m}_{X}(r)-1\right]-r c t}}, \quad t \geq 0
$$

where $R(t)$ is given in (21).

It can be shown that $M$ is an $\mathbf{F}$-martingale where the filtration is given by $\mathcal{F}_{t}=$ $\mathcal{F}_{\infty}^{\Lambda} \vee \mathcal{F}_{t}^{R}$. A lower bound is obtained when $0 \leq t_{0}<\infty$ as

$$
\begin{aligned}
\mathbb{E}^{\mathcal{F}_{0}}\left[M\left(T_{u}\right) \mid T_{u} \leq t_{0}\right] & \geq \mathbb{E}^{\mathcal{F}_{0}}\left[e^{-\Lambda\left(T_{u}\right)\left[\hat{m}_{X}(r)-1\right]+r c T_{u}} \mid T_{u} \leq t_{0}\right] \\
& \geq \inf _{0 \leq t \leq t_{o}} e^{-\Lambda(t)\left[\hat{m}_{X}(r)-1\right]+r c t}
\end{aligned}
$$

More precisely,

$$
\begin{aligned}
\mathbb{E}^{\mathcal{F}_{0}} & {\left[M\left(T_{u}\right) \mid T_{u} \leq t_{0}\right] } \\
& =\mathbb{E}^{\mathcal{F}_{0}}\left[e^{-r\left[u+R\left(T_{u}\right)\right]} e^{-\Lambda\left(T_{u}\right)\left[\hat{m}_{X}(r)-1\right]+r c T_{u}} \mid T_{u} \leq t_{0}\right] \\
& \geq \inf _{0 \leq t \leq t_{o}}\left\{e^{-\Lambda(t)\left[\hat{m}_{X}(r)-1\right]+r c t}\right\} \mathbb{E}^{\mathcal{F}_{0}}\left[e^{-r\left[u+R\left(T_{u}\right)\right]} \mid T_{u} \leq t_{0}\right] \\
& \geq \inf _{0 \leq t \leq t_{o}}\left\{e^{-\Lambda(t)\left[\hat{m}_{X}(r)-1\right]+r c t}\right\} \inf _{y \geq 0}\left\{\frac{1-F_{X}(y)}{\int_{y}^{\infty} e^{-r(y-x)} d F_{X}(x)}\right\} .
\end{aligned}
$$

Then we get, from (38), that

$$
P^{\mathcal{F}_{0}}\left\{T_{u} \leq t_{0}\right\} \leq \frac{M(0)}{\mathbb{E}^{\mathcal{F}_{0}}\left[M\left(T_{u}\right) \mid T_{u} \leq t_{0}\right]} \leq e^{-r u} \sup _{0 \leq t \leq t_{o}} l(r ; t)
$$

Taking expectations proves (24). Using (39) in (40) yields (25). 


\section{References}

[1] Ammeter, H. (1948). "A generalization of the collective theory of risk in regard to fluctuating basic probabilities", Skand. Aktuarietidskr., 31, 171198.

[2] Asmussen, S. (1989). "Risk theory in a Markovian environment", Scandinavian Actuarial Journal, (2), 69-100.

[3] Asmussen, S. and Rolski, T. (1994). "Risk theory in a periodic environment: the Cramér-Lundberg approximation and Lundberg inequality", Mathematics of Operations Research, 19, 410-433.

[4] Beard, R.E., Pentikäinen, T. and Pesonen, E. (1984). Risk Theory. 3rd ed., Chapman \& Hall, London.

[5] Berg, M. and Haberman, S. (1994). "Trend analysis and prediction procedures for time nonhomogeneous claim processes", Insurance: Mathematics and Economics, 14, 19-32.

[6] Björk, T. and Grandell, J. (1988). "Exponential inequalities for ruin probabilities in the Cox case", Scandinavian Actuarial Journal, (1-2), 77-111.

[7] Dassios, A. and Embrechts, P. (1989). "Martingales and insurance risk", Communications in Statistics: Stochastic Models, 5(2), 181-217.

[8] Daykin, C.D., Pentikäinen, T. and Pesonen, M. (1994). Practical Risk Theory for Actuaries. Chapman \& Hall, London.

[9] Dimitrov, B., Chukova, S. and Garrido, J. (2000). "Compound counting processes in a periodic random environment", Journal of Statistical Research, 34(2), 99-111.

[10] Embrechts, P., Grandell, J. and Schmidli, H. (1993). "Finite-time Lundberg inequalities in the Cox case", Scandinavian Actuarial Journal, (1), 17-41.

[11] Garrido, J. and Lu, Y. (2002). "On double periodic non-homogeneous Poisson processes", Working paper 02-43, Business Economic Series, Universidad Carlos III de Madrid.

[12] Grandell, J. (1991). Aspects of Risk Theory. Springer-Verlag, Berlin.

[13] Grandell, J. (1995). "Some remarks on the Ammeter risk process", Mitt. Ver. Schweiz. Vers. Math., 95, 43-72. 
[14] Han, Q. and Aki, S. (1998). "Formulae and recursions for the joint distributions of success runs of several lengths in a two-state Markov chain", Statistics and Probability Letters, 40, 203-214.

[15] Morales, M. (2004) "On a periodic risk averse process: a simulation approach", North American Actuarial Journal, forthcoming.

[16] Rolski, T., Schmidli, H., Schmidt, V. and Teugels, J. (1999). Stochastic Processes for Insurance and Finance, Wiley, New York.

[17] Schmidli, H. (1996). "Lundberg inequalities for a Cox model with a piecewise constant intensity", Journal of Applied Probability, 33(1), 196-210.

[18] Schmidli, H. (1997). "Estimation of the Lundberg coefficient for a Markov modulated risk model", Scandinavian Actuarial Journal, (1), 48-57.

[19] Schmidli, H. (2003). "Modelling PCS options via individual indices", CAF Working Paper, No. 157, University of Aarhus. 


\section{List of Recent Technical Reports}

46. Eduardo Rodriguez, Scoring Methods for Risk Classification, April 2000

47. R. Rodríguez-Ramos, L. Cortés-Carrasquero, J. Bravo-Castillero and R. Guinovart-Díaz, Homogeneization in Elasto-plastic Composites, June 2000

48. A. A. Alvarez-Mesquida, R. Rodríguez-Ramos, F. Comas, R. Esquivel and G. Monsivais, Scattering of Shear Horizontal Elastic Waves in a Heterogeneous Piezoelectric Medium, June 2000

49. M. L. Filshtinsky, R. Rodríguez-Ramos and O. Sanchez-Casals, Fracture Mechanic in Piezoceramic Composite Plate, June 2000

50. F. Lebon, R. Rodriguez-Ramos and A. Mesejo, Homogenization and Wavelet-Galerkin Method for a Nonlinear One-dimensional Problem, June 2000

51. L. Yang, The Impact of Mortality Improvement on Social Security, August 2000

52. Rodrigo Arias López and José Garrido, Bounds and Other Properties of the Inverse, Moments of a Positive Binomial Variate, September 2000

53. B. N. Dimitrov, Z. Khalil, M. E. Ghitany and V. V. Rykov, Likelihood Ratio Test for Almost Lack of Memory Distributions, November 2001

54. Yogendra P. Chaubey and Anthony Crisalli, The Generalized Smoothing Estimator, April 2002

55. Yogendra P. Chaubey and Pranab K. Sen, Smooth Isotonic Estimation of Density, Hazard and MRL Functions, April 2002

56. Pablo Olivares, Maximum Likelihood Estimators for a Branching-Diffusion Process, August 2002

57. Shuanming Li and José Garrido, On Ruin for the Erlang(n) Risk Process, June 2003 
58. G. Jogesh Babu and Yogendra P. Chaubey, Smooth Estimation of a Distribution and Density function on a Hypercube Using Bernstein Polynomials for Dependent Random Vectors, August 2003

59. Shuanming Li and José Garrido, On the Time Value of Ruin for a Sparre Anderson Risk Process Perturbed by Diffusion, November 2003

60. Yogendra P. Chaubey, Cynthia M. DeSouza and Fassil Nebebe, Bayesian Inference for Small Area Estimation under the Inverse Gaussian Model via Cibbs Sampling, December 2003

61. Alexander Melnikov and Victoria Skornyakova, Pricing of EquityLinked Life Insurance Contracts with Flexible Guarantees, May 2004

62. Yi Lu and José Garrido, Regime-Switching Periodic Models for Claim Counts, June 2004.

Copies of technical reports can be requested from:

Prof. Xiaowen Zhou

Department of Mathematics and Statistics

Concordia University

7141, Sherbrooke Street West

Montréal (QC) H4B 1R6 CANADA 